\title{
A rational and iterative process for targeted nanoparticle design and validation
}

\author{
Laura Rodriguez-Lorenzo ${ }^{\mathrm{a}, *}$, Sarah D. Rafiee ${ }^{\mathrm{b}, 1}$, Corine Reis ${ }^{\mathrm{b}, 1}$, Ana Milosevic ${ }^{\mathrm{a}}$, \\ Thomas L. Moore ${ }^{\mathrm{a}}$, Sandor Balog ${ }^{\mathrm{a}}$, Barbara Rothen-Rutishauser ${ }^{\mathrm{a}}$, Curzio Ruegg ${ }^{\mathrm{b}}$, \\ Alke Petri-Fink ${ }^{\mathrm{a}, \mathrm{c}, * *}$
}

a Adolphe Merkle Institute, University of Fribourg, Chemin des Verdiers 4, 1700 Fribourg, Switzerland

b Deparment of Oncology, Microbiology and Immunology, Faculty of Sciences and Medicine, University of Fribourg, Chemin du Musée 18, 1700 Fribourg, Switzerland

${ }^{\mathrm{c}}$ Chemistry Department, Faculty of Sciences and Medicine, University of Fribourg, Chemin du Musée 9, 1700 Fribourg, Switzerland

\section{A R T I C L E I N F O}

\section{Keywords:}

Multifunctional gold nanoparticles

Anti-biofouling

ERBB2-positive breast cancer cells

Trastuzumab

FACS

ICP-OES

DF-HIS

\begin{abstract}
A B S T R A C T
The lack of understanding of fundamental nano-bio interactions, and difficulties in designing particles stable in complex biological environments are major limitations to their translation into biomedical clinical applications. Here we present a multi-parametric approach to fully characterize targeted nanoparticles, and emphasizes the significant effect that each detail in the synthetic process can have on downstream in vitro results. Through an iterative process, particles were designed, synthesized and tested for physico-chemical and bio-interactive properties which allowed the optimization of nanoparticle functionality. Taken together all interative steps demonstrate that we have synthesized a multifunctional gold nanoparticles that can detect ERBB2-positive breast cancer cells while showing stealth-like behavior toward ERBB2-negative cells and excellent physicochemical stability.
\end{abstract}

\section{Introduction}

Nanotechnology has seen a surge in interest over the past two decades. This is most evident in the rise in budget allocations to the National Nanotechnology Initiative (NNI; USA) from \$450 million at its inception in 2001 to approximately \$1.5 billion in 2015 (www.nano. gov), and the European Commission's distribution of approximately €1 billion in grants from 2014 to 2015 as part of the Horizon 2020 program to nanosciences, nanotechnologies, materials and new production technologies (ec.europa.eu/programmes/horizon2020). In the biomedical field this coincides with an increasing number of research publications regarding nanotechnology and nanomedicine [1], excitement regarding the intellectual property potential of biomedical nanoparticles (NPs) [2], potential benefits of NPs to innovatively treat different pathologies, and approval of several nano-formulations for clinical use or investigation in clinical trials [3-7]. Despite the significant excitement, there has been little translation of NPs into actual clinically used products relative to the research output $[1,8]$. This is in part due to the relative infancy of the field, a lack of understanding of fundamental nano-bio interactions at cellular and systemic levels, and difficulty in manufacturing particle systems stable in complex biological environments $[9,10]$. One aspect of biomedical NP development that can be neglected is rational particle design, which is in particular crucial as typically used nano drug carriers are often highly complex engineered materials [11-13]. In a 2013 editorial, Howard \& Peer [11] called for more comprehensive and robust physicochemical and biological characterization, and a recent perspective by Chan and colleagues lamented the inability of nanomedicine research to significantly improve tumor-targeting with NPs [14].

In this proof-of-concept study, we present a conceptual road map for the rational design and synthesis of targeted multifunctional gold nanoparticles (AuNPs), which we developed for breast cancer targeting. Specifically, we aimed to emphasize the necessity for multiple and complementary characterization techniques that can inform critical steps in the particle development process (e.g. particle colloidal stability in complex media and targeting efficacy). Fig. 1 outlines the iterative process undertaken in particle design using a logical decision tree to break down the NP design into the four smaller branches, i.e. (I)

\footnotetext{
* Corresponding author. Current address: International Iberian Nanotechnology Laboratory, Av. Mestre José Veiga s/n, 4715-330, Braga, Portugal.

** Corresponding author at: Adolphe Merkle Institute, University of Fribourg, Chemin des Verdiers 4, 1700 Fribourg, Switzerland.

E-mail addresses: laura.rodriguez-lorenzo@inl.int (L. Rodriguez-Lorenzo), alke.fink@unifr.ch (A. Petri-Fink).

1 These authors contributed equally to the work.
} 


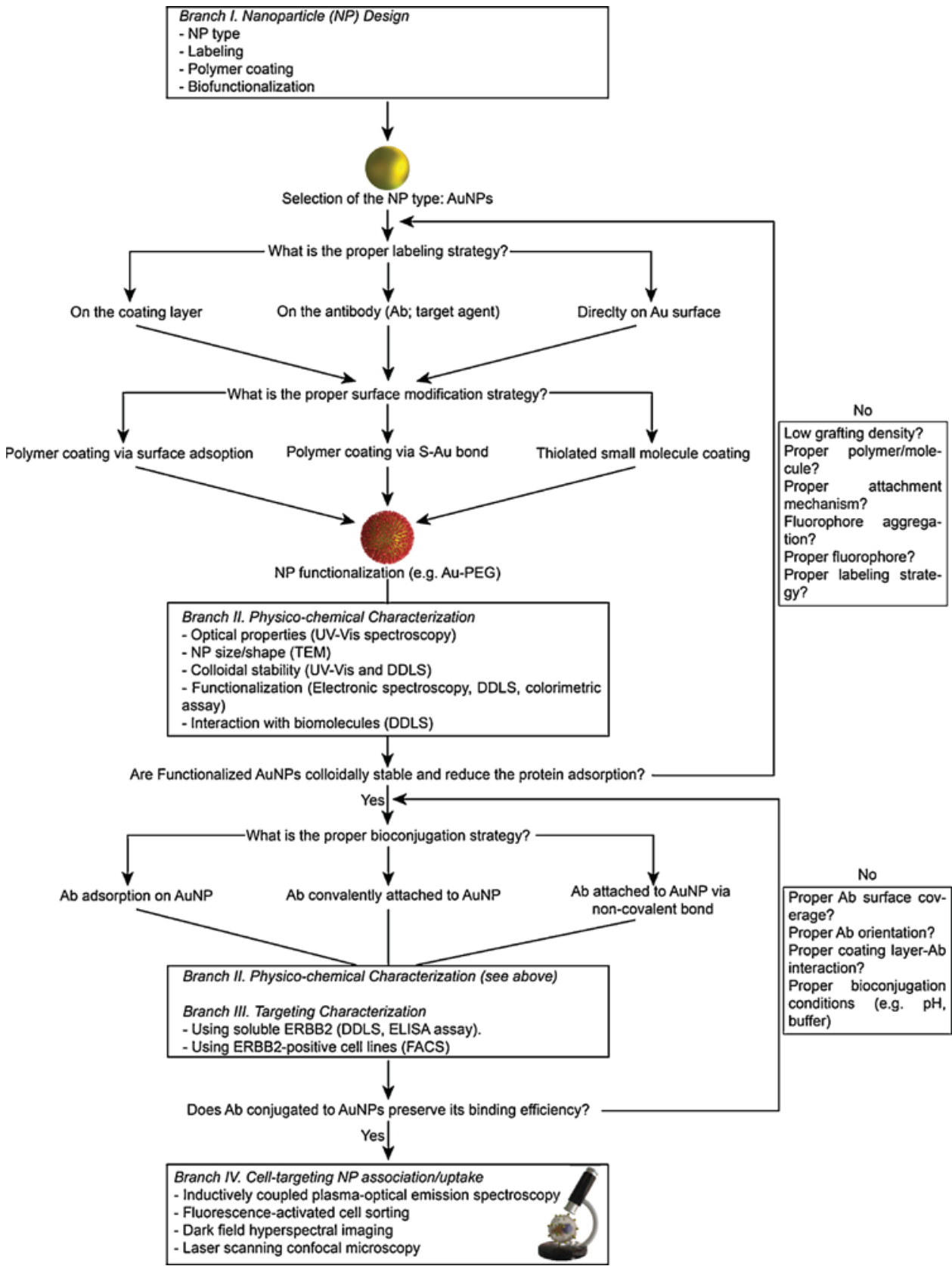

Fig. 1. Decision tree for the design of multifunctional NPs via an integrated approach. In this iterative process, multiple analytical methods (grouped in the decision tree with roman numerals) are used throughout each step of the process to optimize particle design and implementation. The multiple methods are organized in four branches: (I) multifunctional NP design; (II) physicochemical characterization; (III) targeting characterization and (IV) NP uptake/association to breast cancer cells. Here we have focused on the parameter that can directly affect the NP design; however, we should keep in mind that the selection of the cell line/biological factors plays a vital role in this entire diagram. Acronyms in the scheme: PEG: Polyethyleneglycol; DDLS: Depolarized dynamic light scattering; TEM: Transmission electron microscopy; ERBB2: erb-b2 receptor tyrosine kinase 2; ELISA: Enzyme-Linked ImmunoSorbent Assay. Here, the case of targeted AuNPs is used as example of how to raise decision making.

multifunctional NP design; (II) physicochemical characterization; (III) targeting characterization and (IV) NP uptake/association to breast cancer cells. Each branch involves multiple analytical steps to ensure the proper material characterization and in vitro validation in the design and implementation of targeted AuNPs. Such a road map aims to provide a template during the rational design of particles for biomedical applications.

Following the approach presented here, we demonstrate the benefit of applying an integrated analytical approach utilizing iterative feedback during synthesis in order to efficiently achieve engineered AuNP with the desired properties with respect to the intended application.

\section{Results and discussion}

Several design parameters were initially decided such as particle type, polymer coating, fluorophore labeling, and bio-conjugation of a targeting molecule (Fig. 1, branch I). Particle physicochemical characterization was performed using multiple techniques such as determining optical properties using UV-vis spectroscopy, particle size and shape with transmission electron microscopy (TEM), colloidal stability in relevant biological media using depolarized dynamic light scattering (DDLS) and UV-vis, and successful bio-conjugation via colorimetric assay (i.e. Bradford assay), UV-vis and SDS-PAGE with silver nitrate $\left(\mathrm{AgNO}_{3}\right)$ staining (Fig. 1, branch II). This battery of analytical techniques feeds back the particle design - optimizing polymer coating density and colloidal stability, reducing protein adsorption on the NP surface, optimizing antibody surface coverage, and tuning targeting ligand conjugation efficiency. Following these steps and optimizing the NP carrier during this stringent analytical process, significantly improved targeting efficiency of breast cancer cells (as a targeting model) as shown by fluorescence-activated flow cytometry, enzyme-linked immunosorbent assay (ELISA), and DDLS (Fig. 1, branch III). This approach also yielded crucial information vis-à-vis targeting molecule orientation, optimal targeting molecule density, and potential interferences/interactions between fluorophores and targeting molecule efficiency. The latter was then validated using a combination of 


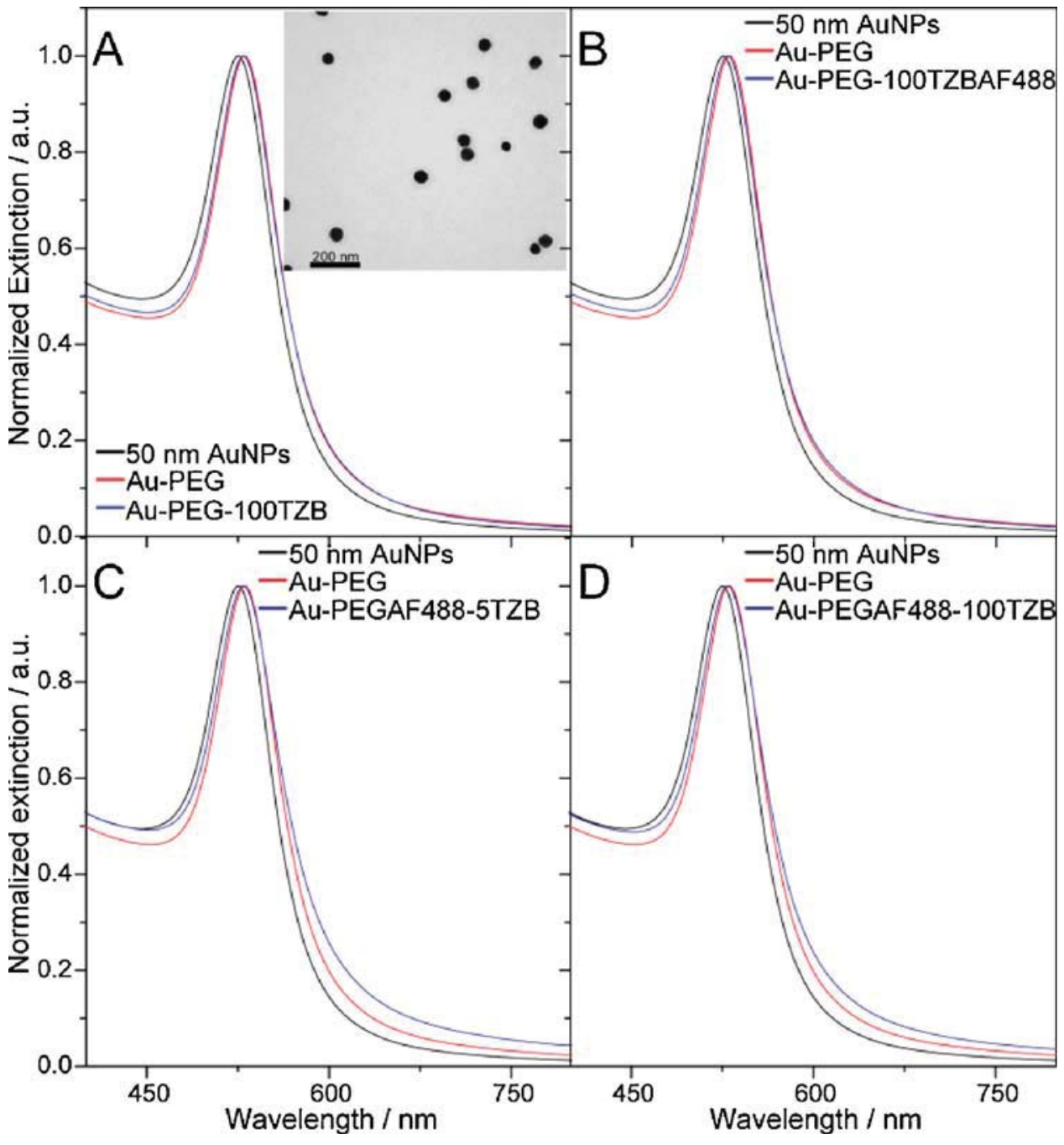

Fig. 2. UV-vis spectra of AuNPs in PBS with varying degrees of functionalization. Bare $50 \mathrm{~nm}$ AuNPs are shown (black lines), as well as PEGylated AuNPs (red lines), and PEGylated AuNPs functionalized with TZB (blue lines) Different panels show PEGylated AuNPs functionalized with $100 \times$ TZB per particle (AuPEG-100TZB) (A), $100 \times$ TZB per particle with AF488 labeled on the TZB molecule (Au-PEG100TZBAF488) (B), PEGylated AuNPs with $5 \times$ TZB per particle with the AF488 dye labeled on the PEG chain (Au-PEGAF488-5TZB) end (C), and PEGylated AuNP with $100 \times$ TZB per particle with the AF488 dye labeled on the PEG chain end (Au-PEGAF488-100TZB). UV-vis spectra were normalized by the extinction maximum. Insert TEM image shows bare $50 \mathrm{~nm}$ AuNP with the scale bar representing $200 \mathrm{~nm}$ (For interpretation of the references to color in this figure legend, the reader is referred to the web version of this article). inductively coupled plasma-optical emission spectrometry (ICP-OES), flow cytometry, dark field hyperspectral imaging (DF-HIS) and laser scanning confocal microscopy (LSM) (Fig. 1, branch IV).

\subsection{Nanoparticle physico-chemical properties and colloidal stability (inter-} connected branches I and II)

We selected AuNPs as a model particle platform to optimize the design of a breast cancer-targeting NP. AuNPs were chosen due to their excellent biocompatibility, ease of synthesis, relatively facile surface functionalization through S-Au bonds, and the associated straightforward analytics. AuNPs with a primary diameter of $50 \mathrm{~nm}$ were selected because they offered a convenient surface area to tune the number of antibodies ( $\mathrm{Ab}$ ) per NP, allowing us to study the possible role of surface coverage on the targeting efficiency of the nanocarrier. The size and shape of the AuNPs were studied by TEM and UV-vis (Fig. 2). TEM results confirmed the size $(51 \pm 7 \mathrm{~nm})$ and spherical shape of the AuNPs. These AuNPs displayed a localized surface plasmon resonance (LSPR) band centered at $527 \mathrm{~nm}$ (Figs. 2 and S1) which corresponds to NPs with a diameter of $46 \mathrm{~nm}$ [15]. AuNPs were functionalized with either heterobifunctional carboxyl-PEG-thiol (COOH-PEG-SH) or with a mixture of COOH-PEG-SH and fluorescently labeled Alexa Fluor 488 (AF488)-PEG-SH (reaction scheme in the Fig. S2A). In the first approach thiolated PEG polymer attaches to the gold surface via the formation of an Au-S bond and allows attachment of biomolecules to the pendant $-\mathrm{COOH}$ group. In the second approach, the mixture of polymers (COOH-PEG-SH and AF488-PEG-SH) provides an optical reporter via the AF488 fluorescent dye and a free $-\mathrm{COOH}$ group for bioconjugation. The PEGylation produced a slight red-shift $(5-6 \mathrm{~nm})$ due to the change in the refractive index from the presence of PEG on Au surface, thus confirming the coating (Figs. 2 and S1) [16].

A non-trivial aspect of biomedical NPs is their colloidal stability in complex biological media. Upon transfer to cell culture media, serum, or blood, particles enter a solution with high ionic strength and are rapidly coated by biomacromolecules. This can cause a number of particulate responses such as particle aggregation, loss of surface coating, or particle dissolution/degradation [10]. Thus, to ensure colloidal stability it is necessary to characterize particles in the appropriate biological media (e.g. cell culture media). The effect of PEG surface coverage on AuNPs stability was tested in cell culture media (Dulbecco's modified Eagle's media, DMEM) supplemented with $10 \%$ fetal calf serum (FCS). UV-vis measurements of AuNPs with a grafting density of $1,5,10,15$, and $30 \mathrm{PEG} / \mathrm{nm}^{2}$ in phosphate buffered saline (PBS) and complete DMEM showed stability for all formulations except $1 \mathrm{PEG} / \mathrm{nm}^{2}$ (Fig. S1). Zeta potential measurements provide insight to this effect; the 5-30 PEG $/ \mathrm{nm}^{2}$ maintained the expected negative surface charge $\left(-12\right.$ to $-19 \mathrm{mV}$, Table S1) due to the carboxylic $\left(-\mathrm{COO}^{-}\right)$ end-group of the PEG chain, however $1 \mathrm{PEG} / \mathrm{nm}^{2}$ was close to neutral $(-5 \pm 6 \mathrm{mV})$. This decrease in the zeta potential may be due to the negatively charged $-\mathrm{COO}^{-}$end group possibly partly embedded in the voids of the PEG layer on the surface [17].

DDLS and zeta potential confirmed the presence of the PEG layer around AuNPs. The results shown in Table 1 reveal that unconjugated PEGylated AuNPs presented higher hydrodynamic radii than the as- 
Table 1

Physicochemical properties of the AuNPs sets.

\begin{tabular}{|c|c|c|c|c|}
\hline Samples & $\begin{array}{l}\text { Hydrodynamic } \\
\text { Radius }^{\mathrm{a}}\left(\mathrm{PD}^{\mathrm{b}}\right) \\
{[\mathrm{nm}]}\end{array}$ & $\begin{array}{l}\text { Zeta } \\
\text { Potential }^{c} \\
( \pm \mathrm{SD}) \\
{[\mathrm{mV}]}\end{array}$ & $\begin{array}{l}\text { AF488/ } \\
\text { AuNP }^{d} \\
( \pm S D)^{\dagger}\end{array}$ & $\begin{array}{l}\mathrm{Ab} / \\
\mathrm{AuNP} \\
( \pm \mathrm{SD})\end{array}$ \\
\hline $\mathrm{Au}$ & $27.5(40.0)$ & $-15.9(1.7)$ & - & - \\
\hline Au-PEG & $59.5(71.6)^{*}$ & $-6.9(1.6)$ & - & - \\
\hline $\begin{array}{l}\text { Au-PEG- } \\
\text { 100TZBAF488 }\end{array}$ & $53.8(81.5)^{*, \S}$ & $-15.3(4.5)$ & 363 (113) & $\begin{array}{l}97 \\
(5)^{*(* *)}\end{array}$ \\
\hline Au-PEG-100TZB & $54.5(82.8)^{*, \S}$ & $-25.8(1.5)$ & - & $101(1)^{*}$ \\
\hline Au-PEGAF488 & $65.6(82.8)^{*}$ & $-11.0(4.6)$ & 417 (225) & - \\
\hline $\begin{array}{c}\text { Au-PEGAF488- } \\
\text { 100TZB }\end{array}$ & $70.5(104.9)^{\$, \S}$ & $-12.0(3.7)$ & $417(225)$ & $100(1)^{*}$ \\
\hline $\begin{array}{l}\text { Au-PEGAF488- } \\
\text { 5TZB }\end{array}$ & $74.7(106.0)^{\ddagger}$ & $-13.5(2.9)$ & 417 (225) & $4(1)$ \\
\hline
\end{tabular}

$*$ and ${ }^{(* *)}$ Indicate a significant difference from Au-PEGAF488-5TZB and AuPEG-100TZB, respectively, as compared by a one-way ANOVA and Turkey's HDS test $(p<0.05)$

a Mean hydrodynamic radius was obtained by DDLS at room temperature and at a scattering angle of $90^{\circ}$. DDLS measurements were carried out in triplicate.

* Indicates a significant difference from $\mathrm{Au}$ as compared by a one-way ANOVA and Turkey's HDS test $(p<0.05)$.

$\S$ Indicates a significant difference from respective PEGylated AuNPs as compared by a one-way ANOVA and Turkey's HDS test $(p<0.05)$.

b Polydispersity (\%).

c Zeta-potential in PBS were measured in 10 runs (mean \pm SD).

d Fluorescence was measured on a VICTOR plate reader with the filter set $560 / 615$.

$\dagger$ No significant difference observed between Au-PEG-100TZBAF488 and the set of Au-PEGAF488 as compared by a one-way ANOVA and Turkey's HDS test $(p<0.05)$.

e Total protein estimation using Branford assay.

synthesized AuNPs: $59.5 \mathrm{~nm}$ for Au-PEG and $65.6 \mathrm{~nm}$ for Au-PEFAF488 vs. $27.5 \mathrm{~nm}$ for non-functionalized AuNPs. The surface grafted PEG chains can acquire either a "brush" or "mushroom" conformation. The latter mainly occurs when distance between the attachment points of PEG to the surface is larger than the Flory radius $\left(\mathrm{R}_{\mathrm{F}}\right)$, while the brush conformation is observed when this distance is smaller than $R_{F}$ [18]. Both PEGylated AuNPs presented a referred distance and grafting coverage of $0.47 \mathrm{~nm}$ and $0.54 \mathrm{PEG} / \mathrm{nm}^{2}$ (Au-PEG) and $0.44 \mathrm{~nm}$ and $0.64 \mathrm{PEG} / \mathrm{nm}^{2}$ (Au-PEGAF488) respectively, as obtained by a method previously described [17]. This indicated that the PEG layers deposited on the AuNPs possessed a brush-like conformation because the $\mathrm{R}_{\mathrm{F}}$ for 5000 Da PEG is $4.9 \mathrm{~nm}$. In contrast, no considerable hydrodynamic size changes were observed upon TZB coupling to any of the PEGylated AuNPs (Table 1), which could be attributed to the compression of neighboring PEG molecules [19].

We qualitatively evaluated the serum protein adsorption levels from the changes in the hydrodynamic radii estimated by DDLS measurements of all AuNPs in PBS buffer and complete DMEM. This method offers scattering information originating exclusively from the NPs on an essentially zero-background [20], which is not achievable using conventional DLS. As shown in Table S1, the size of carboxyl-terminated PEGylated AuNPs remains unchanged under all conditions, suggesting that these particles are not affected by the biomolecules present and remain colloidally stable. This finding is in agreement with UV-vis spectra data in Fig. S1 showing the absence of significant size changes except in the case of the $1 \mathrm{PEG}$ molecule $/ \mathrm{nm}^{2}$ grafting density, where a small shoulder at $600 \mathrm{~nm}$ appears due to a slight aggregation. With these results, we confirmed that the PEG layer acts as an anti-biofouling agent and that all formulations were colloidally stable in the respective environment. In addition, we examined the possibility of PEG layer displacement from the Au surface by measuring the fluorescence intensity of Au-PEGAF488 in PBS vs. fluorescence intensity in supplemented DMEM and FACS buffer after $4 \mathrm{~h}$ dialysis (see details in the SI).
Fig. S3 shows very low fluorescence decay, which proves that no significant PEG displacement is produced.

Thus, the colloidal stability measurements (branch II Fig. 1) have emphasized two important points. Firstly, colloidal stability can be an overlooked aspect of particle design and synthesis. For example, standard DLS to evaluate NP colloidal stability in complex media is insufficient because "zero-background" cannot be achieved in proteinrich media, and therefore require specialized techniques (i.e. DDLS) or advanced expertise to avoid misinterpretation of data. The main reason is that the magnitude of the specific measurement property (i.e. light scattering) of the biomolecules can be of the same order as the NPs, which generates a biased analysis [20]. Particle stability in purified $\mathrm{H}_{2} \mathrm{O}$ does not entail particle stability in more complex solutions. Therefore, multiple robust and sensitive characterization methods are a necessity to glean a complete understanding of NP colloidal stability in complex media. Secondly, NP design parameters such as particle stabilizing molecule surface coverage and "long term" particle stability need to be considered. Having optimized these factors, we could progress to determining cellular targeting efficacy.

\subsection{Breast cancer cells as a relevant model to evaluate nanoparticle targeting efficiency (important factor for branches III and IV)}

Tumor-targeted AuNPs are considered a promising platform for NPbased diagnostics and therapy. Tumor cells often differentially express cell surface molecules (receptors) compared to normal cells. Such receptors can be exploited for targeting, diagnostic and therapy using proteins (e.g. transferrin) [21], small molecules [22], aptamers [23,24], peptides $[25,26]$, or monoclonal antibodies (mAbs) $[27,28]$. However, the use of targeting ligands for particle-mediated diagnostics or therapy is a complicated matter. Generally, particles are envisioned for systemic administration into the bloodstream, and once there a targeted particle must localize and be retained at the site of action. However, particles in circulation will naturally be cleared by the body, must bind to target sites in dynamic and competitive environments, may exhibit off-target binding, and most are aimed at heterogeneous cell populations (and therefore show variable efficacy) [29]. Moreover, there can be shielding of targeting ligands via specific or non-specific protein adsorption (i.e. formation of a protein corona) on the particle surface [30]. In this context, particle targeting must rely on specific optimization of the targeting ligand attachment to the particle, and in-depth characterization of the targeting efficacy.

In order to evaluate the targeting efficiency of our multifunctional AuNPs platform we used three human breast cancer cell lines expressing different levels of the receptor tyrosine-protein kinase erbB-2 (ERBB2), also known as human epidermal growth factor 2 (HER2). ERBB2 is overexpressed in many cancers, including a subtype of breast cancer, compared to normal cells [31]. The anti-ERBB2 antibody Trastuzumab (TZB, humanized IgG1) is therapeutically used in patients with ERBB2-positive tumors to block ERBB2 signaling and has been used experimentally for tumor targeting purposes [32-34]. Thus, we selected TZB as a model mAb for targeting ERBB2-positive breast cancer cells to test the various parameters pertinent to the NP design process.

We used unlabeled free TZB and a secondary fluorescent Ab in a FACS analysis to monitor the expression of ERBB2 in three breast cancer cell lines: BT-474 human breast ductal carcinoma, as well as SKBR-3 and MDA-MB-231 human breast adenocarcinomas. Fig. S4 shows the FACS data and the calculated number of ERBB2 receptors normalized by the cell area of the cells. BT-474 expressed the highest level of ERBB2, SK-BR-3 slightly less and MDA-MB-231 the least. Based on this and the previous confirmation of AuNPs colloidal stability, we then set to develop the ERBB2-targeted AuNPs. 


\subsection{Trastuzumab maintains ERBB2 binding efficiency following} nanoparticle conjugation (Inter-connected branches I, II and III)

To design ERBB2-targeted AuNPs, we conjugated TZB to the carboxylic groups of PEGylated AuNPs through a two-step protocol using a carbodiimide chemistry (Bioconjugation reaction in the Fig. S2B) [35]. We designed four targeted multifunctional AuNPs: (1) Unlabeled PEGylated AuNPs with a full surface TZB coverage (Au-PEG-100TZB), used as control. (2) Unlabeled PEGylated AuNPs with a full surface coverage of TZB directly labeled with AF488 (Au-PEG-100TZBAF488), used to investigate the fluorophore effect on the TZB binding efficiency. (3) AuNPs with AF488-labeled PEG (Ratio 1:1 PEG:PEGAF488) with low surface coverage of TZB (Au-PEGAF488-5TZB), used to examine the effect of TZB surface coverage on the binding efficiency. (4) AuNPs with AF488-labeled PEG (Ratio 1:1 PEG:PEGAF488) with full surface coverage of TZB (Au-PEGAF488-100TZB), used to test the TZB binding efficiency when the fluorophore is on the PEG. Detailed physicochemical properties of the prepared AuNPs are summarized in Table 1.

To validate the successful conjugation of TZB to the AuNPs, AuNPPEG-TZB was analyzed by reducing SDS-PAGE electrophoresis (see details in SI). $\mathrm{AgNO}_{3}$ gel staining showed bands at 50 and $25 \mathrm{kDa}$, confirming the successful conjugation of TZB to the particles (Fig. S5). To determine the average number of TZB molecules attached to each AuNPs, we indirectly quantified non-attached TZB during the bioconjugation step using the Bradford assay. We determined $\mathrm{Ab}$ conjugation was highly efficient (yield $>93 \%$, Table 1 ). The number of AF488 molecules per AuNPs in labeled TZB (Fig. S6) and PEGAF488 AuNPs were similar (Table 1), thereby eliminating bias in the comparison of receptor binding efficiency due to the difference in the fluorescence labeling. The zeta potential values demonstrated that the AuNPs retained their negative surface charge despite of attached TZB. In addition, we assessed colloidal stability of the multifunctional AuNPs in DMEM containing 10\% FCS and FACS buffer (5 mM EDTA, 3\% FCS in PBS) by UV-vis spectroscopy. No large shift and broadening of the LSPR were observed for any of the TZB-conjugated AuNPs, thereby excluding aggregation (Fig. S7) [36].

As the covalent conjugation of TZB on the AuNP surface could impact the TZB binding efficiency to ERBB2 [34], we determined the AuNP-PEG-TZB binding capacity to recombinant ERBB2 extracellular domain (ECD) in two assays (Branch III in Fig. 1): ELISA and DDLS (Fig. 3). To confirm the successful binding of multifunctional AuNPs to soluble ERBB2-ECD, we analyzed the formation of TZB-AuNPs-ERBB2ECD complexes in suspension by monitoring changes in the hydrodynamic size using time-resolved DDLS. The results were compared with Au-PEG particles incubated with ERBB2-ECD. PEGylated AuNPs showed no affinity for the ERBB2-ECD. The anti-human IgG antibodyconjugated AuNPs acted as positive control by non-specifically binding to ERBB2-ECD. Fig. 3A-C displays the results of $2 \mathrm{~h}$ RT kinetic measurements of three different TZB:ERBB2-ECD ratios: 1:1, 1:2 and 1:5 with $1 \times 10^{10}$ AuNPs, which corresponds to $1 \times 10^{12}$ TZB molecules. This incubation time was chosen in accordance with previous FACS and ICP-OES experiments.

When Au-PEG-100TZB was incubated in ERBB2-ECD in aqueous solution, a very rapid response occurred. Even within the first minute, time-resolved DDLS was not able to reveal the initial phase of the TZBAuNPs:ERBB2-ECD interaction. During time we observed an increase of hydrodynamic size for TZB-conjugated AuNPs while no changes were observed for PEG-coated AuNPs. This increase in size is due to the ERBB2-ECD binding to TZB on AuNP resulting in a greater total hydrodynamic radius $\left(R_{h}\right)$. The $\Delta R_{h}$ was found to be $8.3 \mathrm{~nm}$ for a $1: 1$ ratio, $13.2 \mathrm{~nm}$ for a $1: 2$ ratio and $24.7 \mathrm{~nm}$ for a $1: 5$ ratio. As TZB is endowed with two identical antigen-binding sites, it is possible that it binds one or two ERBB2-ECD depending on its concentration. Troise et al. [37] reported that TZB can simultaneously bind two receptor molecules at low concentration, while at high concentration the binding is mainly monovalent. In our case, the concentration of TZB used was $3.3 \mathrm{nM}$, for which the system was most likely in the two-site binding regime. However, the presence of AuNPs most likely affects the antibody-antigen binding kinetics, given that the binding equilibrium is not reached in $2 \mathrm{~h}$. Yin et al. [34] reported that the presence of AuNPs has an effect on binding kinetics of TZB with ERBB2-expressing cells: nanoconjugates exhibited faster association rate constant than free TZB molecules. However, the dissociation rate constants were also much faster for nanoconjugates, resulting in a weaker binding affinity than that of free TZB. This is likely due to the negative charge of TZB-conjugated AuNPs that inhibits its accessibility to the negatively charged cell membrane.

ELISA was performed to determine the ability of TZB-conjugated AuNPs to recognize surface immobilized ERBB2-ECDC (i.e. coated on wells of a 96-well plate). Fig. 3E shows the same trend that with DDLS experiments. Taken together, these results indicate that TZB conjugated to AuNPs retains its affinity for ERBB2-ECD.

\subsection{Specific orientation of Trastuzumab for optimal ERBB2 targeting (Branch III)}

With the chosen conjugation strategy, Abs can be bound to the AuNP in a number of ways and orientations, some of which can interfere with access to the binding site, possibly resulting in a decreased activity of the bound Abs. As an example, the activity of a single chain $\mathrm{Ab}$ fragment against human cancer antigen TAG-72 bound at about a 1:1 $\mathrm{M}$ ratio to PEG is decreased up to $40 \%$ [38]. Even greater reduction has been observed when multiple PEG chains are conjugated to a single $\mathrm{Ab}$ [39]. A directed Ab conjugation strategy helping correct orientation of the $\mathrm{Ab}$ was reported to favorably affect the accessibility of antigen binding sites [40-42]. Therefore, TZB conjugation was carried out by controlling orientation of the $\mathrm{Ab}$ on the NP surface through ionic and covalent interactions [43]. This approach is based on the fact that when the $\mathrm{pH}$ of an $\mathrm{Ab}$ solution is lower than the $\mathrm{Ab}$ isoelectric point, the concentration of positive charges $\left(-\mathrm{NH}^{+}\right.$group of Lys residues) in the Fc region of the $\mathrm{Ab}$ is high. As negatively charged PEGylated AuNPs $\left(\mathrm{COO}^{-}\right.$group of $\left.\mathrm{PEG}\right)$ preferentially interact with the positively charged Fc region of TZB this results in amide bond formation in the Fc region, while leaving the antigen binding sites largely free (Fig. S2B). We chose two TZB surface coverage densities (i.e. full coverage at 100 TZB per AuNPs and low coverage at 5 TZB per AuNPs) to study the effect of the ERBB2 binding efficiency.

We initially investigated if the ratio of PEG:PEGAF488 influenced targeting to BT-474 (high expressing ERBB2, Fig. S4). AuNPs with PEG:PEGAF488 of $1: 100$ or $1: 1$ (with equivalent TZB conjugation) were tested by FACS (Table S2). To increase sensitivity, a secondary IgGAF488 Ab was used. A PEG:PEGAF488 of 1:1 was optimal for BT-474 targeting (Fig. 4A). ERBB2 targeting was further evaluated by comparing TZB linked to the AuNPs via the fixed orientation vs. conventional carbodiimide protocol. Results from these experiments showed better binding when the TZB was conjugated with the fixed orientation method for both $5 \times$ TZB per NP (Fig. 4B) and $100 \times$ TZB per NP (Fig. 4C).

These experiments identified two important factors to consider when optimizing targeted NP design. Firstly, the density of fluorophore can have an important role in the targeting efficiency because their presence can reduce the association of AuNPs-conjugated Abs with their receptor by increasing steric hindrance [44,45]. The optimization of fluorophore density is also important to avoid fluorescence decay when dye molecule aggregates as described previously [46]. Secondly, targeting ligand orientation is also critical to minimizing hindrance of the Fab binding site not only due to directly covalently modification (attaching Ab through Fab to the NP), but also to Fab shielding because of its embedding in the polymer layer [47]. 


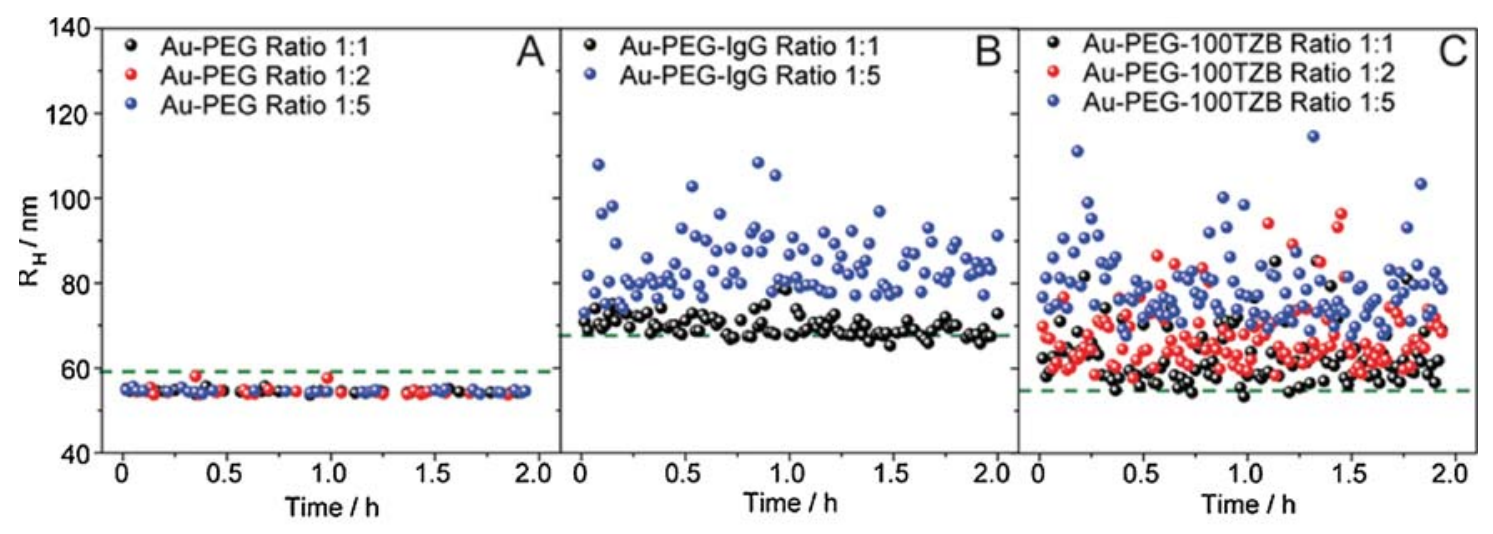

\begin{tabular}{lcccc}
\hline D Sample & \multicolumn{4}{c}{ Hydrodynamic radius [nm] (mean \pm SD) } \\
\cline { 2 - 5 } & PBS & Ratio 1:1 & Ratio 1:2 & Ratio 1:5 \\
\hline Au-PEG & $59.5 \pm 0.3$ & $53.7 \pm 5.1$ & $51.9 \pm 15$ & $54.6 \pm 0.3$ \\
Au-PEG-IgG & $66.9 \pm 2.8$ & $70.0 \pm 2.6$ & Aggr. & $84.7 \pm 7.2^{*}$ \\
Au-PEG-TZB & $54.5 \pm 0.4$ & $62.8 \pm 6.6^{*}$ & $67.7 \pm 7.8^{*}$ & $79.2 \pm 8.5^{*}$ \\
\hline
\end{tabular}

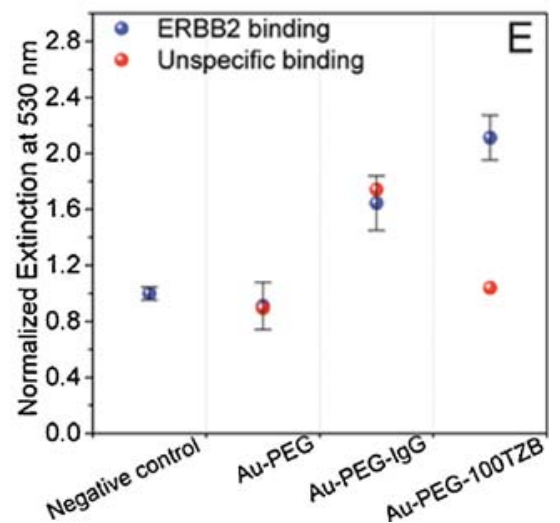

Fig. 3. Time-resolved DDLS study started promptly after incubating AuNP-PEG (A), AuNP-PEG-IgG (B), or AuNP-PEG-TZB (C) in presence of soluble ERBB2-ECD at different concentrations in water (ratio of TZB to ERBB2-ECD). The dashed lines correspond to the hydrodynamic size of AuNPs in PBS buffer. Hydrodynamic radius measurements are summarized in the table (D). * Indicates a significant difference from the respective hydrodynamic size in PBS as compared by a one-way ANOVA and Turkey's HDS test $(p<0.05)$. (E) ELISA assay performed in the presence (blue) and absence (red) of ERBB2-ECD protein verified the specificity of Au-PEG100TZB to ERBB2-ECD. As negative control, a well coated with ERBB2-ECD protein was used. The absorbance was normalized with respect to negative control (For interpretation of the references to color in this figure legend, the reader is referred to the web version of this article).

2.5. Significance of detection method on cell-targeting nanoparticle association (Branch IV)

The specificity of TZB-conjugated AuNPs for ERBB2-positive breast cancer cells was investigated using the three aforementioned human breast cancer cell lines. Different targeted AuNP formulations were incubated with cells for either $2 \mathrm{~h}$ or $24 \mathrm{~h}$, and uptake of AuNPs was determined via ICP-OES and FACS. To verify that there were no adverse effects on cells following NP exposure, cells were first incubated with AuNPs at $40 \mu \mathrm{g} / \mathrm{mL}$. No morphological changes in phase contrast images of living cells (Fig. S8) and excellent viability in the MTT viability assay [48] were observed after $24 \mathrm{~h}$ of AuNPs incubation with the three breast cancer cell lines (Fig. S9).

Cell-specific binding efficiency of targeted multifunctional AuNPs was tested by incubating various AuNPs for $2 \mathrm{~h}$ with the three cell lines followed by FACS analysis. A secondary AF488-conjugated anti-human IgG antibody was added to enhance detection [49]. Specific targeting in ERBB2 positive BT-474 and SK-BR-3 cells was achieved for the four TZB-conjugated multifunctional AuNPs at higher MFI values (Fig. 5A and B). In the case of Au-PEGAF488-100TZB, differences in MFI between MDA-MB-231 (low ERBB2 expression), BT-474 and SK-BR-3 (high ERBB2 expression) were statistically non-significant ( ${ }^{*} p<5$; MDA-MB-231: $2.24 \pm 0.84 ;$ BT-474: $2.68 \pm 0.24$ and SK-BR-3: $4.09 \pm 1.92$ ). This could be attributed to increased non-specific endocytic uptake, which has been observed when the gold surface is coated fully with Abs [33,34]. Thus, discrimination between the BT-474 and SK-BR-3 was achieved only with Au-PEG-100TZB (3.30 \pm 0.14 for BT-474 vs $6.12 \pm 2.73$ for SK-BR-3).

FACS confirmed that none of the non-targeted, PEGylated (no TZB)
AuNPs associated with any of the cancer cell lines (Fig. 5A and B). This result is consistent with a brush conformation of PEG on the Au surface, which mitigates protein adsorption and non-specific endocytosis as reported for dendritic cells [50]. FACS analysis was complemented by ICP-OES analysis allowing direct quantification of gold associated with the cells (Fig. 5C and D). A control experiment (Fig. S10) showed that there was no significant absorption of AuNPs onto well plates in the absence of cells, therefore excluding signals due to AuNPs bound to plastic. In contrast, all TZB-conjugated AuNPs showed cellular association. All four TZB-conjugated AuNPs showed higher binding efficiency for BT-474 and SK-BR-3 cell lines.

We observed some discrepancies between the cellular binding of AuNP detected by FACS (Fig. 5A and B) vs. ICP-OES (Fig. 5C and D). Fig. 5E shows the comparison between FACS and ICP-OES analyses. This qualitative correlation clearly demonstrates the impact of the presence of AF488 and TZB surface coverage on AuNPs binding efficiency. The presence of fluorophore on AuNPs did not significantly impact binding to ERBB2, since the values for Au-PEG-100TZBAF488 (AF488 bound to TZB) and Au-PEGAF488-100TZB (AF488 bound to $\mathrm{PEG}$ ) did not present any significant change compared to control (AuPEG-100TZB). However, a decrease in TZB surface coverage not only had an impact on the NP-cellular association, but also resulted in a worse correlation between the two techniques. This may be due to the fact that while in TZB-conjugated AuNP TZB is the dominant factor driving AuNP-cancer cell association, in the absence of TZB, the fluorophore passive targeting via nonspecific cellular uptake pathways may become more prominent [50]. Therefore, as the number of TZB molecules at the AuNP surface decreases the presence of "non-shielded PEG" on the Au surface increases resulting in decreased nonspecific 

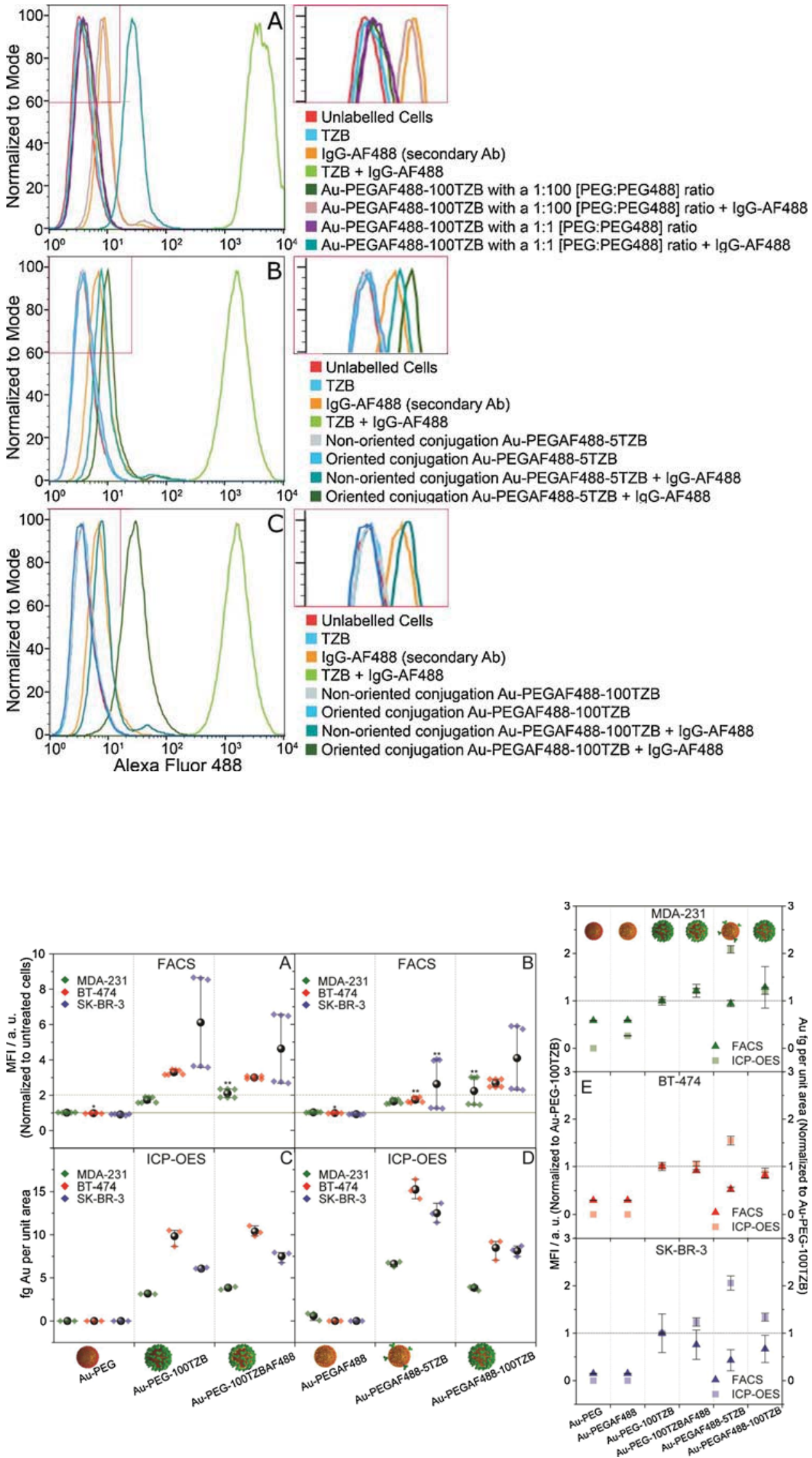

Fig. 5. Cellular association of AuNPs determined via FACS (A, B) and ICP-OES (C, D) after exposure for $2 \mathrm{~h}$. The location of the fluorophore label was tested (i.e. AF488 conjugated to the PEG chain end vs. conjugated to the Fc chain of TZB), as well as the TZB density. In order to compare the two methods, normalized AuNPs association by MDA-MB231 (upper panel, green), BT-474 (middle panel, red) and SK-BR-3 (lower panel, blue) was shown as a function of TZB conjugation and detection techniques, FACS (triangle) and ICP-OES (square). For FACS measurements (A, B), samples were measured in sextuplicate (light green, red and blue diamonds). The black spheres indicate the mean values \pm SD. Solid horizontal line indicates the normalized MFI of untreated cells and dotted horizontal lines indicates the normalized MFI of non-specific staining of AF488-labeled secondary antibody. Krauskal-Wallis and U-Mann-Whitney nonparametric tests were performed to evaluate non-statistically significant differences with untreated cells (*p<0.05) and non-specific staining of secondary antibody $(* * p<0.05)$. For ICP-OES measurements (C, D) each condition was measured in triplicate (semi-transparent diamonds). The black spheres and lines indicate the mean values and SD respectively. Split-plot ANOVA with Huynh-Feldt correction for non-sphericity and Bonferroni's post hoc test were performed to evaluate non-statistically significant differences with Au-PEG and Au-PEGAF488 (* $p<0.05$ ), respectively (For interpretation of the references to color in this figure legend, the reader is referred to the web version of this article). 
uptake. This effect may be relevant for in vivo experiments because reducing the coverage of $\mathrm{Ab}$ on NP will also results in fewer $\mathrm{Fc}$ portions available for binding to $\mathrm{Fc}$ receptors expressed in immune cells [51]. Therefore, optimization of $\mathrm{Ab}$ surface coverage is important to maximize targeting efficiency and minimize non-specific receptor binding and NP clearance. Furthermore, the difference observed between FACS and ICP-OES cell association data, is likely due to the fact that a secondary labeled antibody was required for FACS analysis. Having fewer Fc portions available due to the low amount of TZB on AuNP surface results in a weaker fluorescence enhancement compared to AuNPs with full surface TZB coverage.

\subsection{Targeted imaging (Branch IV)}

To further confirm targeting of the breast cancer cells by the four TZB-conjugated AuNPs, we performed dark field optical microscopy with hyperspectral imaging (DF-HSI). DF-HSI detects light that is elastically scattered by the metallic NPs. It is a powerful tool for imaging at the single-particle level, making it an alternative imaging technique for AuNPs [52]. After $24 \mathrm{~h}$ incubation about 30\% of AuNP were cell associated, compared to $8 \%$ at $2 \mathrm{~h}$ (Fig. S11). We therefore decided to perform DF-HSI analysis at $24 \mathrm{~h}$. Under a dark-field microscope, where only scattered light is collected [53], TZB-conjugated AuNPs could be clearly seen as red/orange dots associated with the cancer cells while no significant differences in scattering were observed in the cells incubated with Au-PEG or Au-PEGAF488 compared to cells alone (Fig. S12). This further confirms that PEGylated AuNPs do not interact with cells. BT-474 and SK-BR-3 cells exposed to TZB-conjugated AuNPs display a more intense scatter than MDA-MD-231 cells, consistent with higher targeted multifunctional AuNPs binding and in agreement with the ICP-OES results (Fig. S11). Therefore, specific ERBB2 targeting was further confirmed (qualitatively) with DF-HSI.

Confocal laser scanning microscopy was attempted to examine the localization and distribution of TZB-conjugated AuNPs with higher precision. A secondary labeled $\mathrm{Ab}$ was used to enhance the fluorescent signal of the TZB (similar to the FACS studies) as it was not possible to image the fluorophores labeled on the AuNPs directly. This is likely due to either self-quenching where emitted photons are reabsorbed by other AF488 fluorophores when AF488 molecules are in close proximity (which is favored for the nanoscale size of AuNPs) [54] or from the nonfluorescent complex formation with amino acids (either free moieties or formed part of protein structure) [55]. BT-474 cells were incubated with NPs at $40 \mu \mathrm{g} / \mathrm{mL}$ for $24 \mathrm{~h}$ or with free TZB. BT-474 cells treated with Au-PEGAF488-100TZB exhibited similar patterns of membrane ERBB2 localization as cells incubated with free TZB (Fig. 6). A previous report showed that ERBB2 receptors undergo endocytosis when bound to TZB adsorbed AuNPs with a diameter of 40 and $50 \mathrm{~nm}$ due to receptor clustering through binding to multivalent AuNPs-bound TZB [56]. However, we found no evidence that Au-PEG-TZB or AuPEGAF488-TZB enhances ERBB2 internalization. This is consistent with the lack of toxicity observed for all TZB-conjugated AuNPs (see MTT assay, Fig. S9), as induction of cell death by TZB involves ERBB2 internalization $[34,54,56]$. Fluorescent signal of the anti-TZB secondary $\mathrm{Ab}$ was noticeably weaker for AuNP-PEGAF488-100TZB compared to free TZB. This is possibly due to steric hindrance as the anti-TZB secondary $\mathrm{Ab}$ binds to the $\mathrm{Fc}$ region of TZB, which in the case of AuNPsPEGAF488-100TZB, is covalently bound to the PEG.

\section{Conclusions}

We demonstrated the benefit of applying an integrated analytical approach utilizing "feedback loops" during the synthesis process in order to efficiently achieve engineered AuNPs. We showed that by using an appropriate process to break down a complex system (fabrication of the optimal targeted nanocarrier) into four smaller packages (I. design; II. physicochemical characterization; III. targeting characterization; IV. NP uptake/association), we can better steer and more assuredly succeed in designing a functional NP with the desired properties respective to the intended application. The outcome of one step may cause a reevaluation of previous decisions. On the contrary, a synthetic approach uncoupled from in-depth analytical considerations may lead to the use of an inappropriate process or waste resources/time because it is unable to break down a complex system into the necessary elements to solve it. As a consequence, the system remains too complex and may fail in delivering the sought after particle without gaining understating for the putative reasons.

Following the decision tree, we designed and established multifunctional AuNPs with requisite characteristics for targeted imaging purposes: (i) A hydrophilic PEG outer shell leading to colloidal stability and reduced nonspecific protein adsorption; (ii) scattering and fluorescent properties provided by the unique optical properties of gold and by the covalent linkage of Alexa Fluor 488 to the PEG, respectively, and (iii) surface functionalization with biologically active ligands that enabled specific targeting. The AuNPs were surface-functionalized with PEG as an anti-fouling agent and conjugated with TZB as an ERBB2targeting NP system. These targeted nanocarriers showed a strong affinity and high specificity to ERBB2-positive cells. These experiments and data show the significance of proper material characterization and in vitro validation in the design and implementation of targeted AuNPs.

\section{Experimental Section}

All glassware was washed with aqua regia and extensively rinsed with water prior to use. Milli-Q grade water was used in all
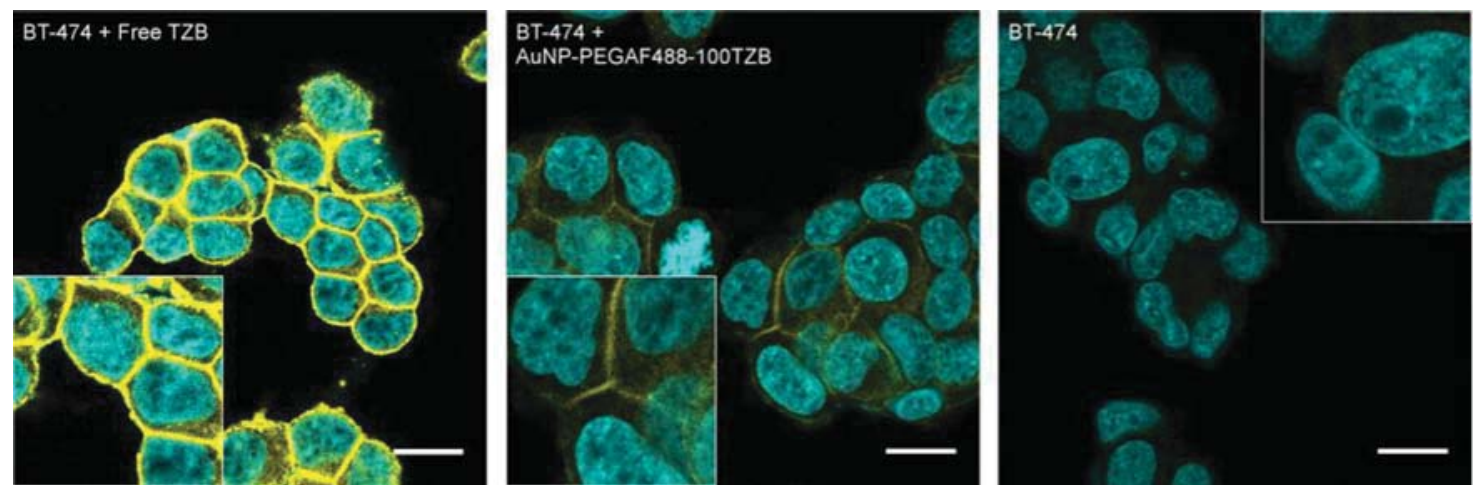

Fig. 6. Laser confocal fluorescence microscopy of BT-474 cells treated for $24 \mathrm{~h}$ with free TZB, Au-PEGAF488-100TZB, and an untreated control. TZB binding was revealed with AF488 labeled secondary IgG antibody (yellow). The nucleus was counterstained with DAPI (cyan). Scale bar is $20 \mu \mathrm{m}$. Both free TZB and AuPEGAF488-100TZB was localized on cellular membrane. Limited NPs-ERBB2 receptor internalization was observed (For interpretation of the references to color in this figure legend, the reader is referred to the web version of this article). 
preparations. All the chemicals were used without further purification.

\subsection{AuNPs synthesis}

Citrate-stabilized AuNPs (20 nm in diameter) were prepared as described [57]. Briefly, a vigorously stirred aqueous tetrachloroauric acid $\left(\mathrm{HAuCl}_{4} \times 3 \mathrm{H}_{2} \mathrm{O} ; 125 \mathrm{~mL}, 0.25 \mathrm{mM}\right.$; Sigma-Aldrich, Buchs, Switzerland) solution was brought to a boil followed by rapid addition of $3 \mathrm{~mL}$ of $40 \mathrm{mM}$ sodium citrate (Sigma-Aldrich). Within $20 \mathrm{~min}$ the color of the solution changed to red, indicating the formation of AuNPs.

$50 \mathrm{~nm}$ AuNPs were synthesized by overgrowth of Au onto the $20 \mathrm{~nm}$ citrate-capped AuNPs (seed dispersion, Fig. S1), as described in Brown et al. [58]. The growth step was carried out by addition of hydroxylamine-hydrochloride $\left(\mathrm{NH}_{2} \mathrm{OH} \cdot \mathrm{HCl} ; 3 \mathrm{~mL}\right.$ of $0.2 \mathrm{M}$; Sigma-Aldrich) and the $20 \mathrm{~nm}$ citrate-capped AuNP dispersion $(15 \mathrm{~mL})$ to a solution of $\mathrm{HAuCl}_{4}(0.25 \mathrm{mM}$ in $270 \mathrm{~mL})$. The resulting AuNPs were washed by centrifugation (4000 rpm, $20 \mathrm{~min}$ ) and re-dispersed in Milli-Q water.

\subsection{AuNPs functionalization with thiolated PEG}

Carbodiimide chemistry was used to conjugate the primary amines of the hetero-bifunctional thiolated PEG (NH2-PEG-SH, MW 5000, Creative PEG-Works, Winston-Salem, NC, USA) with the activated carboxyl groups of the Alexa Fluor 488 NHS ester (AF488 NHS; Thermo Fischer, Reinach, Switzerland), forming an amide functional group as previously described in Rodriguez-Lorenzo et al. [50]. Briefly, $75 \mathrm{mg}$ of polymer, $300 \mu \mathrm{L}$ of 1-ethyl-3-(3-dimethylaminopropyl)-carbodiimide (EDC; Sigma-Aldrich) $(2 \% \mathrm{w} / \mathrm{v}$ EDC) in $\mathrm{pH} 7.4$ phosphate buffered saline 1X (PBS; Life Technologies, Thermo Fischer), and $90 \mu \mathrm{L}$ of Alexa Fluor 488 NHS (AF488 NHS; Invitrogen, Thermo Fischer) solution $(2 \mathrm{mg} / \mathrm{mL}$ in DMF) were mixed on a shaker overnight at room temperature. The conjugated polymer was purified by dialysis and lyophilized for 2 days (stored at $-20^{\circ} \mathrm{C}$ ).

In order to design multifunctional AuNPs, two sets of PEGylated particles were prepared: unlabeled and labeled PEGylated AuNPs. The PEGylated AuNPs sets were prepared by adding either COOH-PEG-SH (Mw 5000, Creative PEG-Works, Winston-Salem, CN, USA) or a mixture of two hetero-functional thiolated PEG [COOHPEG:AF488PEG] at a ratio of 1:1, COOH-PEG-SH for attaching an antibody and AF488-PEG$\mathrm{SH}$ for providing the fluorescence functionality, dropwise to $10 \mathrm{~mL}$ of synthesized AuNPs under shaking to reach a final PEG concentration of 10 PEG molecules $/ \mathrm{nm}^{2}$. The mixture was allowed to react at room temperature protected from light for $24 \mathrm{~h}$. The PEGylated AuNPs were centrifuged (4000 rpm, $20 \mathrm{~min}$ ) twice to remove excess of polymer and re-dispersed in $1 \mathrm{~mL}$ of PBS $1 \times$.

\subsection{Antibody labeling}

Trastuzumab (Herceptin ${ }^{\circ}$ Genentech, South San Francisco, CA), was labeled with AlexaFluor 488 using Alexa Fluor 488 Microscale Protein Labeling Kit (A30006, Life Technologies, Thermo Fischer) according to the provided protocol (Fig. S2C). Briefly, $100 \mu \mathrm{g}$ of Trastuzumab in $100 \mathrm{mM}$ sodium bicarbonate buffer $(\mathrm{pH} 8.3)$ were incubated with an appropriate volume of Alexa Fluor 488 TFP ester solution $(11.3 \mathrm{nmol} / \mu \mathrm{L})$, based on the desired dye:protein molar ratio $(55,35,25$ and 15) for $15 \mathrm{~min}$. Unreacted dye was removed using a size exclusion column provided in the kit. The degree of labeling (DOL) was obtained via the concentration of protein measured by absorbance at $280 \mathrm{~nm}$ (A280) and the concentration of dye measured at an absorbance of $494 \mathrm{~nm}$ (A494) using a NanoDrop ND-1000 spectrophotometer (NanoDrop Technologies, Rockland, Delaware, USA).

\subsection{Antibody conjugation to AuNPs}

The fixed-orientation conjugation of Trastuzumab to PEG-coated AuNPs was carried out as previously reported by Parolo et al. [43] with slight modifications. A solution of 7.4 $\mu \mathrm{L}$ of $\mathrm{N}$-Hydroxysulfosuccinimide sodium salt (sulfo-NHS, Sigma-Aldrich) $(100 \mathrm{mg} / \mathrm{mL})$ and $40 \mu \mathrm{L}$ EDC $(10 \mathrm{mg} / \mathrm{mL})$ was prepared and mixed at room temperature for $10 \mathrm{~min}$. This solution was then mixed with $1 \mathrm{mg}$ of PEGylated AuNPs and the total volume was brought to $1 \mathrm{~mL}$ with MES (Sigma-Aldrich) buffer (10 mM, pH 5). The suspension was incubated at $37^{\circ} \mathrm{C}$ under shaking (650 rpm) for $30 \mathrm{~min}$ to activate the carboxylic groups of the PEG. After the incubation, the conjugate solution was collected by centrifugation ( $4000 \mathrm{rpm}$ ) for $5 \mathrm{~min}$ and was mixed with $1 \mu \mathrm{g}$ or $20 \mu \mathrm{g}$ of TZB depending on the desired number of TZB molecules per AuNPs ( 5 or 100 TZB, respectively). The solution was diluted to a final volume of $1 \mathrm{~mL}$ in MES buffer. The supernatant containing the excess EDC-sulfo-NHS was collected for quantification of unbound TZB by the Bradford assay. The diluted suspension of TZB-conjugated AuNPs was then incubated at $37^{\circ} \mathrm{C}$ under shaking for $60 \mathrm{~min}$. The obtained conjugates were centrifuged at $4000 \mathrm{rpm}$ at $4{ }^{\circ} \mathrm{C}$ for $20 \mathrm{~min}$ and then re-dispersed with $1 \mathrm{~mL}$ of sodium bicarbonate buffer $(10 \mathrm{mM}, \mathrm{pH} 8)$ at $37^{\circ} \mathrm{C}$ for $30 \mathrm{~min}$ under shaking. The AuNPs were then centrifuged $(4000 \mathrm{rpm})$ at $4{ }^{\circ} \mathrm{C}$ for $20 \mathrm{~min}$ and the remaining activated carboxyl group was blocked with $40 \mathrm{mM}$ ethanolamine (Sigma-Aldrich) at $\mathrm{pH}$ 6. The resulting suspension was then incubated at $37^{\circ} \mathrm{C}$ under shaking $(650 \mathrm{rpm})$ for $60 \mathrm{~min}$. Finally, the resulting oriented-TZB-conjugated AuNPs were centrifuged (4000 rpm for $20 \mathrm{~min}$ ) and re-dispersed in $1 \mathrm{~mL}$ PBS $1 \times$.

\subsection{Characterization methods}

Optical characterization was carried out by UV-vis spectra of the samples at $25^{\circ} \mathrm{C}$ using a Jasco V-670 spectrophotometer (Jasco Europe S.R.L., Milano, Italy), using $10 \mathrm{~mm}$ path length quartz cuvettes. The UV-vis spectra were acquired in water, PBS 1X, Dulbecco's modified Eagle medium (DMEM GlutaMAX ${ }^{\mathrm{TM}}$ ) cell culture medium supplemented with $10 \%$ fetal calf serum (FCS), and FACS buffer to assess the colloidal stability. The particle size was determined directly from UV-vis spectra using the tabulated theoretical data of uncoated spherical AuNPs in water described in Haiss et al. [15] Micrographs of the AuNPs were taken with a Tecnai Spirit transmission electron microscope (FEI, Frankfurt, Germany), operating at $120 \mathrm{kV}$. The images were recorded at a resolution of $2048 \times 2048$ pixels (Veleta CCD camera, Olympus, Volketswil, Switzerland). Fluorescence intensity was measured using a fluorescence plate reader with excitation wavelength centered at $485 \mathrm{~nm}$ and emission filter centered at $535 \mathrm{~nm}$ (Wallac 1420 VICTOR3 reader, PerkinElmer, Boston, MA), using appropriate band pass filters. The surface charge of AuNP samples was measured in suspension of $50 \mu \mathrm{g} / \mathrm{mL}$ in PBS $1 \times$ at $25^{\circ} \mathrm{C}$ using a phase amplitude light scattering (PALS) zeta potential analyzer (Brookhaven Instruments Corporation, Hotsville, NY, USA). The Smoluchowski approximation [59] was fitted to 10 cycles of electrophoretic mobility (EPM) measurements and 10 replicates were obtained for each sample to estimate the mean and standard deviation (SD). Hydrodynamic size was measured by DDLS (details about DDLS theory in SI). Samples were diluted into PBS $1 \times$ and complete phenol red-free DMEM and measurements were carried out at $25^{\circ} \mathrm{C}$ with a scattering angle of $90^{\circ}$. DDLS spectra were collected using a commercial goniometer instrument (LS instruments AG, Fribourg, Switzerland) equipped with a $500 \mathrm{~mW}$ diode pumped solid-state laser $(660 \mathrm{~nm})$. Data was collected over $6 \mathrm{~min}$ and three independent correlation functions were measured. Further explanation of light scattering measurements can be found in the supplementary information.

The antibody conjugation yield was determined using the Bradford protein concentration assay for both conjugation strategies [60]. After conjugation, the AuNPs with TZB were centrifuged and the supernatant was dialyzed against water to carry out the assay. A reference solution was prepared having exactly the initial TZB concentration under the same conditions. Therefore, the decrease in TZB concentration in the supernatant can be directly correlated to the amount of antibody conjugated on the AuNPs. The AF488 amount on the AuNPs was 
determined by fluorescence using a Perkin Elmer Victor Microplate Reader (560/615 filter set). $10 \mu \mathrm{L}$ of AuNPs samples were dissolved with $10 \mu \mathrm{L}$ of $40 \mathrm{mM}$ KCN (Sigma Aldrich) to avoid any interference during the fluorescence measurements.

\subsection{Trastuzumab (TZB) binding activity by time-resolved depolarized dynamic light scattering (DDLS)}

DDLS measurements were performed to determine the ability of TZB-conjugated AuNPs to recognize soluble ErbB2 receptor. Time-resolved DDLS, we were able to monitor changes in the hydrodynamic radius of AuNPs when the TZB-ERBB2-ECD protein complex is formed. In the DDLS experimental set-up, TZB-conjugated AuNPs and ERBB2ECD protein were free in solution at three TZB:ERBB2 ratios: 1:1 $\left(1.10 \times 10^{12}: 1.10 \times 10^{12}\right), 1: 2\left(1.10 \times 10^{12}: 2.20 \times 10^{12}\right)$ and $1: 5$ $\left(1.10 \times 10^{12}: 5.50 \times 10^{12}\right)$. Time-resolved studies were carried out for Au-PEG, Au-PEG-100TZB, and Au-PEG-100IgG at a gold concentration of $0.15 \mathrm{mM}$.

\subsection{Enzyme-linked immunosorbent assay (ELISA)}

The affinity of AuNP-PEG-TZB for isolated ERBB2-ECD protein was measured by direct ELISA assay as described in Troise et al. [37]. Briefly a 96-well plate was coated with $62.6 \mathrm{nM}$ of ERBB2-ECD protein, at $0.358 \mu$ g protein per well in $100 \mu \mathrm{L}$ miliQ water, kept overnight at $4{ }^{\circ} \mathrm{C}$ and blocked for $2 \mathrm{~h}$ at $25^{\circ} \mathrm{C}$ with $1 \%$ Bovine Serum Albumin (BSA, Sigma-Aldrich, Buchs, Switzerland). After blocking, the Au-PEG, AuPEG-100TZB and Au-PEG-IgG were incubated with a ratio 1:10 (AuNPs: ERBB2-ECD). Binding was determined by measuring the absorption from gold at $525 \mathrm{~nm}$ (corresponding to LSPR of AuNPs) using an ELISA microplate spectrophotometer (ELISA Benchmark Plus, BIORAD, Segrate, Italy).

\subsection{Cell culture}

Human breast cancer cell lines SK-BR-3, BT-474 (high ERBB2 expression), and MDA-MB-231 (low ERBB2 expression) were used in this study [61]. Cells were cultured in DMEM supplemented with $10 \%$ FCS, $100 \mathrm{U} / \mathrm{mL}$ Penicillin and $100 \mu \mathrm{g} / \mathrm{mL}$ Streptomycin at $37^{\circ} \mathrm{C}$ in $5 \% \mathrm{CO}$. All cell culture reagents were purchased from Life Technologies (Thermo Fisher, Carlsbad, CA, USA).

\subsection{Inductively coupled plasma optical emission spectroscopy}

Cells were seeded at $2.5 \times 10^{5}$ cells per well in 24-well plates. Cells were allowed to adhere overnight, and the next day media was removed and cells were exposed to AuNPs at concentration of $40 \mu \mathrm{g} / \mathrm{mL}$. Cells were incubated with NPs for $2 \mathrm{~h}$ and $24 \mathrm{~h}$, and then washed twice with PBS $1 \times$. Cell-associated gold was measured by ICP-OES (Optima 7000DV, Perkin Elmer). Cells were treated with a 2:1 mixture of $65 \%$ $\mathrm{HNO}_{3}$ (Sigma-Aldrich) and 30\% $\mathrm{H}_{2} \mathrm{O}_{2}$ (Reacto Lab SA, Servion, Switzerland) at $300 \mu \mathrm{L}$ per sample and digested for $4 \mathrm{~h}$. The samples were briefly sonicated at $50^{\circ} \mathrm{C}$ prior to the addition of $400 \mu \mathrm{L}$ of $37 \%$ $\mathrm{HCl}$ (Sigma-Aldrich) and digested overnight. Finally, samples were collected in $15 \mathrm{~mL}$ tubes and diluted ten-fold with Milli-Q water. ICPOES measurements were performed at a wavelength of $242.795 \mathrm{~nm}$ (limit of detection (LOD) $=16 \mu \mathrm{g} / \mathrm{L}$ and limit of quantification $(\mathrm{LOQ})=23 \mu \mathrm{g} / \mathrm{L}$ ), at an axial plasma view. The plasma flow was $15 \mathrm{~mL} / \mathrm{min}$ and the sample flow rate $1.5 \mathrm{~mL} / \mathrm{min}$. A washing-step was performed between each measurement and each sample was measured three times. Sample means were compared with a Split-plot ANOVA with Huynh-Feldt correction for non-sphericity $(p<0.05)$ and Bonferroni's post hoc test.

\subsection{Flow cytometry analysis}

Cells were cultured overnight, harvested with trypsin $1 \times$ (Trypsin $10 \times$ diluted in PBS $1 \times$; Sigma-Aldrich) and $2.5 \times 10^{5}$ cells were transferred to $5 \mathrm{~mL}$ tubes. Cells were washed with FACS buffer $(5 \mathrm{mM}$ ethylenediaminetetraacetic acid (EDTA), 3\% FCS in PBS) by centrifuging ( $1400 \mathrm{rpm}$ at $4{ }^{\circ} \mathrm{C}$ for $5 \mathrm{~min}$ ) and re-suspending in $100 \mu \mathrm{L}$ FACS buffer containing $40 \mu \mathrm{g} / \mathrm{mL}$ of AuNPs. Incubation was done at room temperature for $2 \mathrm{~h}$, with gentle agitation. Untreated cells were used as negative control while cells stained with $2 \mu \mathrm{g} / \mathrm{mL}$ of Trastuzumab and $20 \mu \mathrm{g} / \mathrm{mL}$ secondary antibody (Alexa Fluor 488 goat anti-human IgG, Life Technologies) were used as positive control. Cells were then washed and re-suspended in $250 \mu \mathrm{L}$ of FACS buffer. Each sample was examined in BD FACSCalibur and the sample measurements and data analysis were performed with FlowJo Software (Ashland, OR, USA). Each FACS analysis was repeated 6 times. Sample means were compared with Krauskal-Wallis and U-Mann-Whitney non-parametric tests $(p<0.05)$.

\section{Associated content}

Supporting Information. Additional information of: labeling and conjugation reactions; UV-vis spectra, Zeta potentials and hydrodynamic radii of AuNPs with different surface coverage of PEG; flow cytometry analysis of labeled TZB, AuNPs with different PEG:PEGAF488 ratio, TZB-conjugated AuNPs using oriented and nonoriented conjugation; SDS-PAGE analysis of Trastuzumab and Au-PEGTZB samples; Colloidal stability of multifunctional AuNPs; Study of AuPEG-100TZB affinity for ERBB2 receptor by ELISA and time-resolved DDLS; Contrast phase images of SK-BR-3, BT-474 and MDA-MD-231; MTT assay; ICP-OES of non-specific adsorption of AuNPs on well plate. This material is available free of charge via the Internet.

\section{Acknowledgements}

This work was supported by the National Centre of Competence in Research Bioinspired Material, the Swiss National Science Foundation, the Adolphe Merkle Foundation and the University of Fribourg. We would like to thank Dr. Miguel Spuch-Calvar for preparing the 3D graphics used in this manuscript.

\section{Appendix A. Supplementary data}

Supplementary data associated with this article can be found, in the online version, at https://doi.org/10.1016/j.colsurfb.2018.07.066.

\section{References}

[1] V.J. Venditto, F.C. Szoka, Cancer nanomedicines: so many papers and so few drugs!, Adv. Drug Deliv. Rev. 65 (2013) 80-88.

[2] V. Wagner, A. Dullaart, A.-K. Bock, A. Zweck, The emerging nanomedicine landscape.pdf, Nat. Biotechnol. 24 (2006) 1211-1217.

[3] M.R. Green, G.M. Manikhas, S. Orlov, B. Afanasyev, a M. Makhson, P. Bhar, M.J. Hawkins, Abraxane, a novel Cremophorfree, albumin-bound particle form of paclitaxel for the treatment of advanced non-small-cell lung cancer, Ann. Oncol. 17 (2006) 1263-1268.

[4] M.E. Davis, Design and development of IT-101, a cyclodextrin-containing polymer conjugate of camptothecin, Adv. Drug Deliv. Rev. 61 (2009) 1189-1192.

[5] Y.C. Barenholz, Doxil ${ }^{\circledR}$ - the first FDA-approved nano-drug: lessons learned, J. Control. Release 160 (2012) 117-134.

[6] J. Hrkach, D. Von Hoff, M.M. Ali, E. Andrianova, J. Auer, T. Campbell, D. De Witt, M. Figa, M. Figueiredo, A. Horhota, Preclinical development and clinical translation of a PSMA-targeted docetaxel nanoparticle with a differentiated pharmacological profile, Sci. Transl. Med. 4 (2012) 128ra39-128ra39.

[7] E. Phillips, O. Penate-Medina, P.B. Zanzonico, R.D. Carvajal, P. Mohan, Y. Ye, J. Humm, M. Gönen, H. Kalaigian, H. Schöder, Clinical translation of an ultrasmall inorganic optical-PET imaging nanoparticle probe, Sci. Transl. Med. 6 (2014) 260ra149-260ra149.

[8] S. Tinkle, S.E. McNeil, S. Mühlebach, R. Bawa, G. Borchard, Y.C. Barenholz, L. Tamarkin, N. Desai, Nanomedicines: addressing the scientific and regulatory gap, Ann. N. Y. Acad. Sci. 1313 (2014) 35-56. 
[9] N. Kamaly, Z. Xiao, P.M. Valencia, A.F. Radovic-Moreno, O.C. Farokhzad, Targeted polymeric therapeutic nanoparticles: design, development and clinical translation, Chem. Soc. Rev. 41 (2012) 2971-3010.

[10] T.L. Moore, L. Rodriguez-Lorenzo, V. Hirsch, S. Balog, D. Urban, C. Jud, B. RothenRutishauser, M. Lattuada, A. Petri-Fink, Nanoparticle colloidal stability in cell culture media and impact on cellular interactions, Chem. Soc. Rev. 44 (2015).

[11] K.A. Howard, D. Peer, Providing the full picture: a mandate for standardizing nanoparticle-based drug delivery, Nanomedicine 8 (2013) 1031-1033.

[12] H.F. Krug, Nanosafety research—are we on the right track? Angew. Chemie Int. Ed. 53 (2014) 12304-12319.

[13] M. Hofmann-Amtenbrink, D.W. Grainger, H. Hofmann, Nanoparticles in medicine: current challenges facing inorganic nanoparticle toxicity assessments and standardizations, Nanomed. Nanotechnol. Biol. Med. 11 (2015) 1689-1694.

[14] S. Wilhelm, A.J. Tavares, Q. Dai, S. Ohta, J. Audet, H.F. Dvorak, W.C.W. Chan, Analysis of nanoparticle delivery to tumours, Nat. Rev. Mater. 1 (2016) 16014.

[15] W. Haiss, N.T.K. Thanh, J. Aveyard, D.G. Fernig, Determination of size and concentration of gold nanoparticles from UV - Vis spectra, Anal. Chem. 79 (2007) $4215-4221$.

[16] S.V. Jenkins, A. Srivatsan, K.Y. Reynolds, F. Gao, Y. Zhang, C.D. Heyes, R.K. Pandey, J. Chen, Understanding the interactions between porphyrin-containing photosensitizers and polymer-coated nanoparticles in model biological environments, J. Colloid Interface Sci. 461 (2016) 225-231.

[17] M. Uz, V. Bulmus, S. Alsoy Altinkaya, Effect of PEG grafting density and hydrodynamic volume on gold nanoparticle-cell interactions: an investigation on cell cycle, apoptosis, and DNA damage, Langmuir 32 (2016) 5997-6009.

[18] K. Rahme, L. Chen, R.G. Hobbs, M.A. Morris, C. O'Driscoll, J.D. Holmes, PEGylated gold nanoparticles: polymer quantification as a function of PEG lengths and nanoparticle dimensions, RSC Adv. 3 (2013) 6085-6094.

[19] B. Farruggia, B. Nerli, G. Picó, Study of the serum albumin-polyethyleneglycol interaction to predict the protein partitioning in aqueous two-phase systems, $\mathrm{J}$. Chromatogr. B. 798 (2003) 25-33.

[20] S. Balog, L. Rodriguez-Lorenzo, C.A. Monnier, M. Obiols-Rabasa, B. RothenRutishauser, P. Schurtenberger, A. Petri-Fink, Characterizing nanoparticles in complex biological media and physiological fluids with depolarized dynamic light scattering, Nanoscale 7 (2015) 5991-5997.

[21] J. Wang, S. Tian, R.A. Petros, M.E. Napier, J.M. DeSimone, The complex role of multivalency in nanoparticles targeting the transferrin receptor for cancer therapies, J. Am. Chem. Soc. 132 (2010) 11306-11313.

[22] R. Weissleder, K. Kelly, E.Y. Sun, T. Shtatland, L. Josephson, Cell-specific targeting of nanoparticles by multivalent attachment of small molecules, Nat. Biotechnol. 23 (2005) 1418-1423.

[23] O.C. Farokhzad, J. Cheng, B.A. Teply, I. Sherifi, S. Jon, P.W. Kantoff, J.P. Richie, R. Langer, Targeted nanoparticle-aptamer bioconjugates for cancer chemotherapy in vivo, Proc. Natl. Acad. Sci. 103 (2006) 6315-6320.

[24] S.E. Baek, K.H. Lee, Y.S. Park, D.-K. Oh, S. Oh, K.-S. Kim, D.-E. Kim, RNA aptamerconjugated liposome as an efficient anticancer drug delivery vehicle targeting cancer cells in vivo, J. Control. Release 196 (2014) 234-242.

[25] F.M. Mickler, L. Möckl, N. Ruthardt, M. Ogris, E. Wagner, C. Bräuchle, Tuning nanoparticle uptake: live-cell imaging reveals two distinct endocytosis mechanism mediated by natural and artificial EGFR targeting ligand, Nano Lett. 12 (2012) 3417-3423.

[26] D. Xiao, H.-Z. Jia, N. Ma, R.-X. Zhuo, X.-Z. Zhang, A redox-responsive mesoporous silica nanoparticle capped with amphiphilic peptides by self-assembly for cancer targeting drug delivery, Nanoscale 7 (2015) 10071-10077.

[27] M.K. Yu, J. Park, S. Jon, Targeting strategies for multifunctional nanoparticles in cancer imaging and therapy, Theranostics 2 (2012) 3-44.

[28] J. Albanell, J. Baselga, Trastuzumab, a humanized anti-HER2 monoclonal antibody, for the treatment of breast cancer, Drugs Today 35 (1999) 931-946.

[29] G.T. Tietjen, L.G. Bracaglia, W.M. Saltzman, J.S. Pober, Focus on fundamentals: achieving effective nanoparticle targeting, Trends Mol. Med. 24 (2018) 598-606.

[30] A. Salvati, A.S. Pitek, M.P. Monopoli, K. Prapainop, F.B. Bombelli, D.R. Hristov, P.M. Kelly, C. Åberg, E. Mahon, K.A. Dawson, Transferrin-functionalized nanoparticles lose their targeting capabilities when a biomolecule corona adsorbs on the surface, Nat. Nanotechnol. 8 (2013) 137-143.

[31] M. Harries, I. Smith, The development and clinical use of trastuzumab (Herceptin), Endocr. Relat. Cancer 9 (2002) 75-85.

[32] J.F. Hainfeld, M.J. O'connor, F.A. Dilmanian, D.N. Slatkin, D.J. Adams, H.M. Smilowitz, Micro-CT enables microlocalisation and quantification of Her2targeted gold nanoparticles within tumour regions, Br. J. Radiol. 84 (2011) 526-533.

[33] I. Steinhauser, B. Spänkuch, K. Strebhardt, K. Langer, Trastuzumab-modified nanoparticles: optimisation of preparation and uptake in cancer cells, Biomaterials 27 (2006) 4975-4983.

[34] L. Yin, Y. Yang, S. Wang, W. Wang, S. Zhang, N. Tao, Measuring binding kinetics of antibody-conjugated gold nanoparticles with intact cells, Small 11 (2015) 3782-3788.

[35] G.T. Hermanson, Bioconjugate Techniques, 3rd ed., Elsevier Inc., London, UK, 2013.

[36] V. Hirsch, C. Kinnear, L. Rodriguez-Lorenzo, C.A. Monnier, B. Rothen-Rutishauser,
S. Balog, A. Petri-Fink, In vitro dosimetry of agglomerates, Nanoscale 6 (2014) 7325-7331.

[37] F. Troise, V. Cafaro, C. Giancola, G. D’Alessio, C. De Lorenzo, Differential binding of human immunoagents and Herceptin to the ErbB2 receptor, FEBS J. 275 (2008) 4967-4979.

[38] L.S. Lee, C. Conover, C. Shi, M. Whitlow, D. Filpula, Prolonged circulating lives of single-chain Fv proteins conjugated with polyethylene glycol: a comparison of conjugation chemistries and compounds, Bioconjug. Chem. 10 (1999) 973-981.

[39] K. Kitamura, T. Takahashi, T. Yamaguchi, A. Noguchi, A. Noguchi, K. Takashina, H. Tsurumi, M. Inagake, T. Toyokuni, S. Hakomori, Chemical engineering of the monoclonal antibody A7 by polyethylene glycol for targeting cancer chemotherapy, Cancer Res. 51 (1991) 4310-4315.

[40] S. Puertas, M. Moros, R. Fernández-Pacheco, M.R. Ibarra, V. Grazú, J.M. De La Fuente, Designing novel nano-immunoassays: antibody orientation versus sensitivity, J. Phys. D Appl. Phys. 43 (2010) 474012.

[41] M. Marciello, M. Filice, D. Olea, M. Velez, J.M. Guisan, C. Mateo, Useful oriented immobilization of antibodies on chimeric magnetic particles: direct correlation of biomacromolecule orientation with biological activity by AFM studies, Langmuir 30 (2014) 15022-15030.

[42] J. Conde, J. Dias, V. Grazú, M. Moros, P. Baptista, J. De La Fuente, Revisiting 30 years of biofunctionalization and surface chemistry of inorganic nanoparticles for nanomedicine, Front. Chem. 2 (2014) 48.

[43] C. Parolo, A. de la Escosura-Muñiz, E. Polo, V. Grazú, J.M. De La Fuente, A. Merkoçi, Design, preparation, and evaluation of a fixed-orientation antibody/ gold-nanoparticle conjugate as an immunosensing label, ACS Appl. Mater. Interfaces 5 (2013) 10753-10759.

[44] U. Resch-Genger, M. Grabolle, S. Cavaliere-Jaricot, R. Nitschke, T. Nann, Quantum dots versus organic dyes as fluorescent labels, Nat. Methods 5 (2008) 763-775.

[45] S. Vira, E. Mekhedov, G. Humphrey, P.S. Blank, Fluorescent-labeled antibodies: balancing functionality and degree of labeling, Anal. Biochem. 402 (2010) $146-150$.

[46] J.B. Randolph, A.S. Waggoner, Stability, specificity and fluorescence brightness of multiply-labeled fluorescent DNA probes, Nucleic Acids Res. 25 (1997) 2923-2929.

[47] L.J. Cruz, P.J. Tacken, R. Fokkink, C.G. Figdor, The influence of PEG chain length and targeting moiety on antibody-mediated delivery of nanoparticle vaccines to human dendritic cells, Biomaterials 32 (2011) 6791-6803.

[48] T. Mosmann, Rapid colorimetric assay for cellular growth and survival: application to proliferation and cytotoxicity assays, J. Immunol. Methods 65 (1983) 55-63.

[49] A. Johannesson, D. Bates, Amplification by second enzymes, in: D.M. Kemeny, S.J. Challacombe (Eds.), ELISA Other Solid Phase Immunoassays Theor. Pract. Asp. John Wiley \& Sons, Cambridge, 1988, p. 378.

[50] L. Rodriguez-Lorenzo, K. Fytianos, F. Blank, C. Von Garnier, B. Rothen-Rutishauser, A. Petri-Fink, Fluorescence-encoded gold nanoparticles: library design and modulation of cellular uptake into dendritic cells, Small 10 (2014) 1341-1350.

[51] H. Duan, S. Nie, Cell-penetrating quantum dots based on multivalent and endosome-disrupting surface coatings, J. Am. Chem. Soc. 129 (2007) 3333-3338.

[52] S. Patskovsky, E. Bergeron, M. Meunier, Hyperspectral darkfield microscopy of PEGylated gold nanoparticles targeting CD44-expressing cancer cells, J. Biophotonics 8 (2015) 162-167.

[53] M. Hu, C. Novo, A. Funston, H. Wang, H. Staleva, S. Zou, P. Mulvaney, Y. Xia, G.V. Hartland, Dark-field microscopy studies of single metal nanoparticles: understanding the factors that influence the linewidth of the localized surface plasmon resonance, J. Mater. Chem. 18 (2008) 1949-1960.

[54] S. Barua, J.-W. Yoo, P. Kolhar, A. Wakankar, Y.R. Gokarn, S. Mitragotri, Particle shape enhances specificity of antibody-displaying nanoparticles, Proc. Natl. Acad. Sci. 110 (2013) 3270-3275.

[55] H. Chen, S.S. Ahsan, M.B. Santiago-Berrios, H.D. Abruña, W.W. Webb, Mechanisms of quenching of Alexa Fluorophores by natural amino acids, J. Am. Chem. Soc. 132 (2010) 7244-7245.

[56] W. Jiang, B.Y.S. Kim, J.T. Rutka, W.C.W. Chan, Nanoparticle-mediated cellular response is size-dependent, Nat. Nanotechnol. 3 (2008) 145.

[57] G. Frens, Controlled nucleation for the regulation of the particle size in monodisperse gold suspensions, Nat. Phys. Sci. 241 (1973) 20-22.

[58] K.R. Brown, D.G. Walter, M.J. Natan, Seeding of colloidal Au nanoparticle solutions. 2. Improved control of particle size and shape, Chem. Mater. 12 (2000) $306-313$.

[59] M. Smoluchowski, Zur Theorie der elektrischen Kataphorese und der Oberflächenleitung [A theory concerning the electric cataphoresis and the surface conduction], J. Phys. Theor. Appl. 6 (1907) 659-660.

[60] M.M. Bradford, A rapid and sensitive method for the quanti-transition could be observed in the second cycle of the DSC tation of microgram quantities of protein utilizing the principle of measurements. Parts of the protein that are essential for its en-protein-dye binding, Anal. Biochem. 72 (1976) 248254.

[61] R.S. Finn, J. Dering, D. Conklin, O. Kalous, D.J. Cohen, A.J. Desai, C. Ginther, M. Atefi, I. Chen, C. Fowst, G. Los, D.J. Slamon, PD 0332991, a selective cyclin D kinase 4/6 inhibitor, preferentially inhibits proliferation of luminal estrogen receptor-positive human breast cancer cell lines in vitro, Breast Cancer Res. 11 (2009) R77. 